\title{
Effect of hydrogen on surface roughening during Si homoepitaxial growth
}

\author{
D. P. Adams ${ }^{\text {a) }}$ and S. M. Yalisove \\ Department of Materials Science and Engineering, University of Michigan, Ann Arbor, \\ Michigan 48109-2136 \\ D. J. Eaglesham \\ AT\&T Bell Laboratories, 600 Mountain Avenue, Murray Hill, New Jersey 07974
}

(Received 26 May 1993; accepted for publication 12 October 1993)

\begin{abstract}
Hydrogen is shown to have a strong influence on the evolution of surface morphology during low temperature $\left(310^{\circ} \mathrm{C}\right) \mathrm{Si}(100)$ homoepitaxy. Molecular beam epitaxy growth in the presence of deuterium shows a surface roughness within the epitaxial film that increases rapidly until the Si film exhibits a crystalline to amorphous transition. The rate at which the surface roughens depends critically on the partial pressure of deuterium. Although the kinetics of growth are sensitive to small pressures $\left(4 \times 10^{-8}\right.$ Torr) of $\mathrm{D}$, it appears that the breakdown of epitaxy does not result from a "critical" D concentration at the surface. This work suggests that the crystalline to amorphous transition, instead, results from increased roughening during epitaxy.
\end{abstract}

Low temperature $\left(<500^{\circ} \mathrm{C}\right) \mathrm{Si}$ molecular beam epitaxy (MBE) is of potential interest for the development of low temperature processes and for novel device structures which consist of monolayer doping profiles. ${ }^{1}$ Utilization of low temperature growth, however, requires a complete understanding of the kinetics of Si layer growth and its relation to film microstructure. In the past, a great deal of work has been directed at understanding Si adatom diffusion on clean, reconstructed (100) surfaces. Both theoretical and experimental studies have been used to determine the activation energies for $\mathrm{Si}$ adatom diffusion along different directions. ${ }^{2,3}$ However, it was not until recently that the microstructure of $\mathrm{Si}(100)$ films grown by $\mathrm{MBE}$ at low temperatures was investigated and correctly characterized in terms of a crystalline to amorphous transition. ${ }^{4}$ Initially, $\mathrm{Si}$ forms as single-crystal material, but at a critical thickness, designated the epitaxial height $\left(h_{\text {epi }}\right)$, the epitaxial layer turns amorphous. Defining $h_{\mathrm{epi}}$ as the midpoint of the crystalline to amorphous transition, the epitaxial thickness was shown to increase with substrate temperature in an Arrhenius fashion $\left(E_{\mathrm{act}}=0.5 \mathrm{eV}\right) .{ }^{4}$ However, the mechanism(s) responsible for the breakdown of epitaxy were not fully understood. ${ }^{5,6}$ In the present study, a more complete examination of the epitaxial thickness phenomenon includes the effects of impurities and the evolution of surface morphology. It is clear that hydrogen can play a strong role in determining the epitaxial thickness. More recently, $h_{\text {epi }}$ was shown to decrease with increasing $H$ partial pressure following a relation, $h_{\text {epi }}=h_{\mathrm{0}}-k \ln P_{\mathrm{H}}\left(P_{\mathrm{H}}\right.$ is the partial pressure of hydrogen). ${ }^{5}$ This showed, e.g., that the presence of only $10^{-8}$. Torr of hydrogen reduced the thickness to breakdown by a factor of 2 for a substrate temperature of $300^{\circ} \mathrm{C}$. In this letter, we show that the kinetic effect of hydrogen during low temperature $\mathrm{Si}$ epitaxy (MBE) is to increase surface roughness. The change in surface morphology during low temperature growth is

") Work done, in part, at AT\&T Bell Laboratories, 600 Mountain Ave., Murray Hill, NJ 07974. monitored for a range of $\mathrm{H}$ partial pressures.

In this experiment, $\mathrm{Si}$ was grown on $\mathrm{Si}(100)$ substrates in a MBE apparatus (base pressures $<1 \times 10^{-9}$ Torr). Substrates were cleaned, prior to loading, using a buffered (dilute, 100:1) HF dip and then placed into the chamber apparatus. After thorough outgassing, approximately 450Å-thick Si buffer layers were grown $\left(T=660^{\circ} \mathrm{C}\right)$ in order to bury residual impurities. Substrates were then cooled quickly and equilibrated at the growth temperature. One substrate temperature $\left(310^{\circ} \mathrm{C} \pm 25^{\circ} \mathrm{C}\right.$, calibrated using an optical pyrometer and thermocouple) was used for low temperature growth. Silicon was deposited using an electron beam evaporator at a single rate of $1 \AA / \mathrm{s}$, while a deuterium source, consisting of an UHV leak valve, a Ta tube, and a hot $\left(\sim 1850^{\circ} \mathrm{C}\right)$ tungsten filament located approximately $6 \mathrm{in}$. from the Si substrate (with line of sight to the wafer surface) was used to dose the Si surface continuously during low temperature growth. Deuterium is used instead of hydrogen because the two are easily distinguished using secondary ion mass spectrometry (SIMS). Also, the thermal desorption and reaction kinetics of deuterium at silicon surfaces have been studied in detail and are similar to those of hydrogen. ${ }^{7}$ Homoepitaxial growth of $\mathrm{Si}(100)$ at $310^{\circ} \mathrm{C}$, without deliberate introduction of deuterium, was conducted only after allowing the vacuum system to recover for long periods of time after closing the $\mathrm{D}$ source. Each deposition with deuterium dosing involved a single, constant deuterium partial pressure. The effects of different amounts of $\mathbf{D}$ on the microstructure were studied using a range $\left(10^{-8}-10^{-6}\right.$ Torr $)$ of $D$ partial pressures.

Germanium marker layers [1/4 monolayer (ML), calibrated using Rutherford backscattering spectrometry] were used to mark the Si surface at different stages of growth. Marker layers were deposited during very brief interruptions at approximately $125 \AA$ intervals of $\mathrm{Si}$ growth. Cross-sectional transmission electron microscopy (TEM) samples were prepared by mechanical thinning to $50 \mu \mathrm{m}$, followed by ion milling ( $3 \mathrm{kV} \mathrm{Ar}$ ) to perforation. Cross-section TEM was used to distinguish the Ge markers, tilting only in the plane of the Si surface around the 


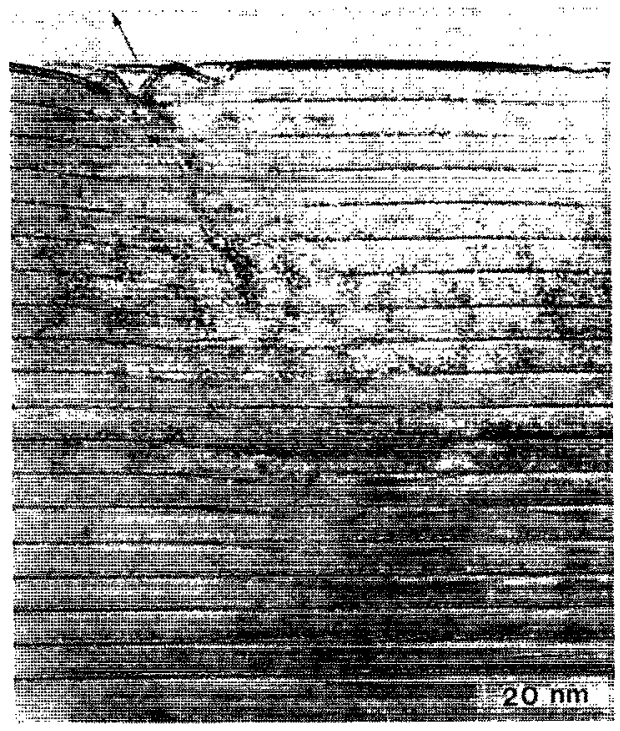

FIG. 1. Cross-section TEM image of a Si layer grown at $310^{\circ} \mathrm{C}$ under "clean" UHV conditions (ambient hydrogen partial pressure). Ge marker layers (1/4 ML) show little change in surface roughness.

$\langle 110\rangle$. A 400 symmetric three-beam image was used for all measurements. Careful control experiments showed that the spread in Gc marker contrast was not due to $D$ enhanced diffusion, but instead was representative of the roughness of the Si surface. ${ }^{8}$ Microdensitometry was used to quantify the roughness of the surface by scanning directly from TEM micrographs. Measured roughness (herein referred to as surface width) at a particular film thickness was taken to be the full width at half-maximum of the spread in the Ge marker layer. Surface roughness was characterized by comparing linescans over small and large $(1 \mu \mathrm{m})$ regions of all micrographs to ensure an accurate measurement, and the magnitude (in $\AA$ ) of the surface width was calibrated using the fringe spacing of the $\mathrm{Si}$ lattice. In addition, control experiments have shown that thin Ge layers do not change the epitaxial thickness of $\mathrm{Si}$ grown at low temperatures. ${ }^{4}$

Figure 1 shows a cross-sectional TEM image of a $\mathrm{Si}$ film grown at low temperature without deliberately introducing atomic deuterium. Growth was terminated at a thickness of $2200 \AA$ (before the crystallinc to amorphous transition). Ge marker layers spaced at $125 \AA$ intervals show that the surface width did not change appreciably over several thousands of $\AA$. In fact, for this film, only small changes in the spread of the Ge marker layer could be detected using TEM. In contrast, very iocalized (not often found on thick buffer layers grown at high temperatures, marked with an arrow in Fig. 1) areas of initially rough ( $\sim 75 \AA$ at the buffer layer surface) material exhibited a different scaling behavior. The surface width increased dramatically. After $2200 \AA$ of epitaxial growth, the roughness in the localized region was approximately 200 $\AA$. Growth characterized by a rapid roughening rate is quite interesting, for such areas were the first to exhibit a breakdown in epitaxy.
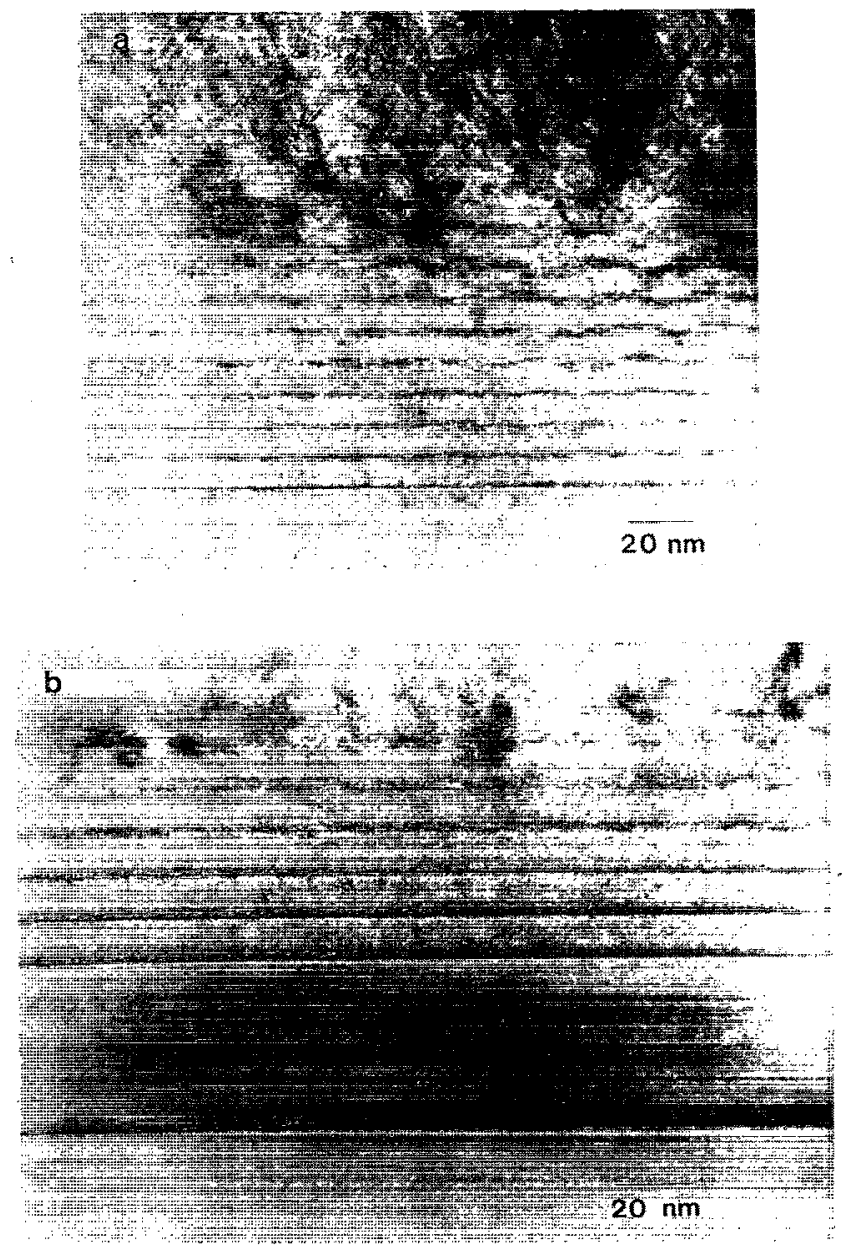

FIG. 2. TEM images of Si layers grown at $310^{\circ} \mathrm{C}$ under a partial pressure of (a) $1.5 \times 10^{-7}$ Torr and (b) $5.0 \times 10^{-7}$ Torr, respectively. Ge layers mark the increase in roughness before the breakdown of epitaxy.

Figure 2 shows the effects of $\mathrm{D}$ partial pressures of $1 \times 10^{-7}$ Torr (estimated to be $\sim 10^{-4} \mathrm{ML} / \mathrm{s}$ from SIMS) ${ }^{5}$ and $5 \times 10^{-7}$ Torr during $\mathrm{Si} \mathrm{MBE}$, at a temperature identical to that in Fig. 1. As can be seen from the three micrographs, the presence of deuterium dramatically changed the surface morphology of the Si layer by increasing the surface roughening rate. For each of the films grown in the presence of deuterium, the surface width increased to approximately $30-50 \AA$ before exhibiting a breakdown in epitaxy. The effects of a range of $D$ partial pressures are shown in Fig. 3. Surface roughness scaled sharply with film thickness for the entire range of pressures tested. Curve fits suggest that a power law dependence of surface width $(w)$ as a function of thickness $(t), w=\alpha t^{\beta}$, most closely matched the data if the roughening exponent, $\beta$, is greater than 1. It was, however, difficult to distinguish between a power law (perhaps $\beta=2$ ) and an exponential as a best fit for the various eurves. It was also indicated in the micrographs that the larger partial pressures of $D$ lowered the epitaxial thickness, $h_{\text {epi }}$. $h_{\text {epi }}$ was measured to decrease with increasing $\mathrm{D}$ partial pressure, following the previously determined relation, $h_{\text {epi }}=h_{0}-k \ln P_{\mathrm{D}}\left(P_{\mathrm{D}}\right.$ is the partial pressure of deuterium). 


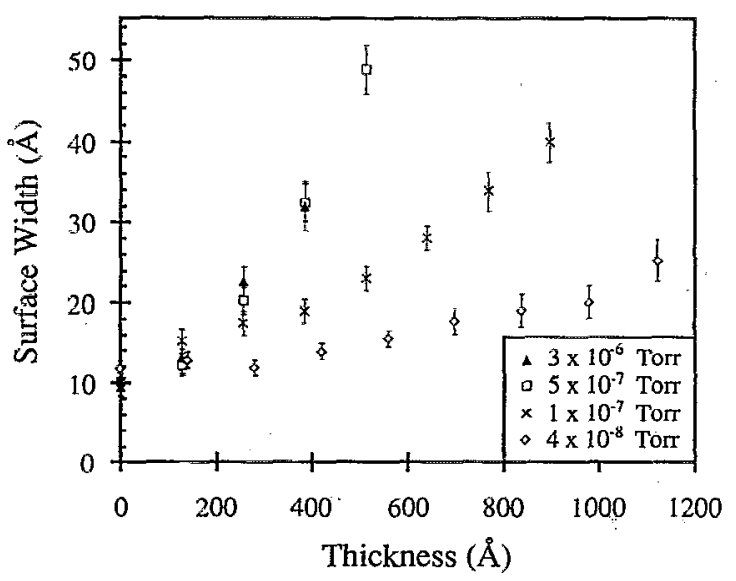

FIG. 3. Surface roughness developed during Si MBE in the presence of different deuterium partial pressures.

This work confirms that hydrogen has a strong influence on $h_{\text {epi }}$ but demonstrates that the effect of hydrogen during low temperature growth is to increase the roughness before breakdown. Past work showed that the pressure dependence of $h_{\text {epi }}$ could not be explained by a threshold $\mathbf{H}$ concentration (via segregation) and suggested that the crystalline to amorphous transition was therefore more likely due to some intrinsic property of low temperature growth. ${ }^{5}$ Since the roughness was shown to scale in a consistent manner with the decrease in $h_{\text {epi }}$ with increasing amounts of deuterium, it is most likely that the breakdown of epitaxy occurs as a result of roughening. Additional evidence supports the model in which a large surface roughness intrinsically leads to the crystalline to amorphous transition. ${ }^{9}$ Growth under clean, UHV conditions showed that regions of the film exhibiting large roughening rates (relative to initially smooth arcas of the surface) were the first to exhibit the crystalline to amorphous transition.

The preliminary roughening data (shown in Fig. 3) for growth in the presence of deuterium is markedly different than that predicted by theory. Several models have been used to predict the dynamic evolution of amplitude fluctuations (surface roughness) for a growing interface. ${ }^{10-12}$ The surface width, $w$, is thought to follow a power law dependence with layer thickness, $t$ (i.e., $w=\alpha t^{\beta}$ ). It is estimated that for the case of limited, yet nonzero diffusion, the roughening exponent, $\beta$, is equal to $0.22-0.25,{ }^{13,14}$ although it is expected that the details of the surface (e.g., defect concentration) may change this slightly. ${ }^{15}$ In the ballistic regime (zero diffusion limit), $\beta$ is thought to be 0.50 , and the morphology is described by a random deposition model. ${ }^{16}$ Nevertheless, only a small amount of experimental work has explored kinetic roughening behavior during thin film growth ${ }^{17}$ According to Kardar, Parisi, and Zhang ${ }^{11}$ and others, ${ }^{18}$ the deposition of atoms onto an initially clean, flat surface should lead to a roughness that increases very slowly with thickness, $\beta \sim 0.3$. The roughening rates suggested by this work more closely follows a nonlinear relation in which $\beta$ is greater than 1 . In addition, if increasing the $D$ partial pressure is changing the kinetics by reducing the Si surface diffusion length, then according to theory, $\beta$ should approach 0.5 (ballistic regime) ${ }^{16}$ As shown in Fig. 3, the roughening rate does not approach this zero diffusion limit but tends to increase with $\beta$ much grcater than 0.5 . Further study will be necessary to precisely determine the role of hydrogen in changing the kinetics during $\mathrm{Si}$ layer growth. It would not be surprising if hydrogen in some way plays a role in limiting surface diffusion lengths, as atomic $\mathrm{H}$ readily bonds with the dimerized $\mathrm{Si}(100)$ surface. ${ }^{19}$ The role of hydrogen in low temperature MBE may be explained by a scenario in which $H$ acts as a barrier to diffusion while saturating the Si dangling bonds.

In summary, this study has shown that the presence of small amounts of hydrogen, during low temperature $\mathrm{Si}$ MBE, strongly affects the film microstructure by increasing the surface roughness. Si films roughen more rapidly when grown in the presence of larger doses of $D$. The surface roughness of each film became large (up to $50 \AA$ ) before exhibiting the crystalline to amorphous transition. This evidence supports a model in which the crystalline to amorphous transition is a result of increased roughening within the epitaxial portion of the film. In addition, the roughening rate of $\mathrm{Si}$ in the presence of deuterium does not follow that predicted by various models on clean surfaces. Surface roughness scales with a superlinear dependence with film thickness.

This work was supported by the NSF under Contract No. DMR 9202176.

${ }^{1}$ Heteroepitaxy in Silicon II, edited by J. C. C. Fan, J. M. Phillips, and B.-Y. Tsaur (Materials Research Society, Pittsburgh, PA, 1987), Vol. 91.

${ }^{2}$ C. Roland and G. H. Gilmer, Phys. Rev. B 46, 13428 (1992).

${ }^{3}$ Y. W. Mo, J. Kleiner, M. B. Webb, and M. G. Lagally, Phys. Rev. Lett. 66, 1998 (1991).

${ }^{4}$ D. J. Eaglesham, H.-J. Gossmann, and M. Cerullo, Phys. Rev. Lett. 65, 1227 (1990).

${ }^{5}$ D. J. Eaglesham, F. C. Unterwald, G. S. Higashi, H. Luftman, D. P. Adams, and S. M. Yalisove (unpublished).

${ }^{6}$ M. V. Murty and H. Atwater (unpublished).

${ }^{7}$ K. Sinniah, M. G. Sherman, L. B. Lewis, W. H. Weinberg, J. T. Yates, and K. C. Janda, J. Chem. Phys. 92, 5700 (1990).

${ }^{8}$ D. P. Adams, D. J. Eaglesham, and S. M. Yalisove, Mater. Res. Soc. Symp. Proc. (to be published).

${ }^{9}$ D. J. Eaglesham and G. Gilmer, in Surface Disordering: Growth, Roughening and Phase Transitions, edited by R. Jullien, J. Kertesz, P. Meakin, and D. E. Wolf (Nova, New York, 1993).

${ }^{10} \mathrm{~J}$. Krug and H. Spohn, in Solids Far from Equilibrium: Growth, Morphology and Defects, cdited by C. Godreche (Cambridge University Press, New York, 1990).

${ }^{11}$ M. Kardar, G. Parisi, and Y.-C. Zhang, Phys. Rev. Lett. 56, 889 (1986).

${ }_{12}$ J. Villain, J. Phys. I 1, 19 (1991).

${ }^{13}$ J. M. Kim and J. M. Kosterlitz, Phys. Rev. Lett. 62, 2289 (1989).

${ }^{14}$ D. E. Wolf and J. Kertesz, Europhys. Lett. 4, 651 (1987).

${ }^{15}$ D. Kandel and D. Mukamel, Europhys. Lett. 20, 325 (1992).

${ }^{16} \mathrm{~J}$. D. Weeks, G. H. Gilmer, and K. A. Jackson, J. Chem. Phys. 65, 712 (1976).

${ }^{17}$ J. Chevrier, V. Le Thanh, R. Buys, and J. Derrien, Europhys. Lett. 16, 737 (1991).

${ }^{18}$ L.-H. Tang, B. M. Forrest, and D. E. Wolf, Phys. Rev. A 45, 7162 (1992).

${ }^{19}$ J. A. Appelbaum and D. R. Hamann, in Theory of Chemisorption, edited by J. R. Smith, Topics in Current Physics (Springer, Berlin, 1980), Vol. 19, p. 43. 